\title{
Associations between Cytokine Levels and CYP3A4 Phenotype in Patients with Rheumatoid Arthritis ${ }^{\mathbb{S}}$
}

\author{
Birgit M. Wollmann, Silje Watterdal Syversen, Maria Vistnes, Elisabeth Lie, Lise L. Mehus, \\ and Espen Molden
}

Center for Psychopharmacology (B.M.W., E.M.), Departments of Rheumatology (S.W.S., E.L.), Internal Medicine (M.V.), and Medicinal Biochemistry (L.L.M.), Diakonhjemmet Hospital, Oslo, Norway; Institute for Experimental Medicinal Research, Oslo University Hospital, Oslo, Norway (M.V.); and Department of Pharmaceutical Biosciences, School of Pharmacy, University of Oslo, Oslo, Norway (E.M.)

Received April 16, 2018; accepted July 9, 2018

\section{ABSTRACT}

Systemic inflammation has been linked to suppressed CYP3A4 activity. The aim of this study was to examine associations between levels of a broad selection of cytokines and CYP3A4 phenotype in patients with rheumatoid arthritis (RA). The study included 31 RA patients treated with tumor necrosis factor (TNF)- $\alpha$ inhibitors. CYP3A4 phenotype was measured as serum concentration of $4 \beta$-hydroxycholesterol $(4 \beta \mathrm{OHC})$ by ultra-performance liquid chromatography-tandem mass spectrometry in samples collected prior to and 3 months after initiation of treatment with TNF- $\alpha$ inhibitors. Serum levels of the following 21 cytokines were determined in the same samples using a beadbased multiplex immunoassay (Luminex technology): CCL2, CCL3, CXCL8, granulocyte colony-stimulating factor, granulocytemacrophage colony-stimulating factor, interferon $\gamma$, interleukin (IL)-1及, IL-1 receptor antagonist (ra), IL-2, IL-4, IL-5, IL-6, IL-7,
IL-10, IL-12, IL-13, IL-15, IL-17A, IL-18, IL-23, and TNF- $\alpha$. Correlations between levels of cytokines and $4 \beta O H C$ were assessed by Spearman's rank correlation tests. Among the investigated cytokines, three were negatively correlated with CYP3A4 phenotype during treatment with TNF- $\alpha$ inhibitors: i.e., IL-1ra $(r=-0.408, P=$ 0.023), IL-6 $(r=-0.410, P=0.022)$ and CXCL8 $(r=-0.403, P=0.025)$ ( $P \geq 0.3$ for all other cytokines). None of the analyzed cytokines were correlated with CYP3A4 phenotype prior to TNF- $\alpha$ inhibitor treatment ( $P>0.1$ for all cytokines). These findings suggest that immune responses associated with increased levels of IL-1ra, IL-6, and CXCL8 may suppress CYP3A4 metabolism. Further studies are required to evaluate these preliminary findings in different patient populations and also examine the possible molecular mechanisms behind our observations.

\section{Introduction}

CYP3A4 is abundantly expressed in human liver and intestine, and is generally regarded as the most important enzyme in drug metabolism (Wilkinson, 2005). There is large interindividual variability in CYP3A4 phenotype (Wilkinson, 2005), as reflected by a $>10$-fold range in enzyme expression in biopsies of human liver and intestine (Ulvestad et al., 2013) and clearance of CYP3A4 probe substrate midazolam (He et al., 2005). The extensive interindividual variability in CYP3A4mediated metabolism is attributed to a combination of genetic and environmental factors (Klein and Zanger, 2013; Hole et al., 2017); however, the clinical relevance of $C Y P 3 A 4$ genetics seems to be limited, and nongenetic factors are likely the most important determinant of the interpatient differences in CYP3A4-mediated metabolism (Klein and Zanger, 2013)

Systemic inflammation has been shown to downregulate the expression of multiple cytochrome P450 (P450) enzymes, and has attracted great interest as a mechanism associated with suppressed CYP3A4 phenotype in patients (Christensen and Hermann, 2012). A number of clinical studies have demonstrated suppressed CYP3A4 phenotype during inflammatory conditions (Mayo et al., 2000; Rivory et al., 2002;

https://doi.org/10.1124/dmd.118.082065.

S This article has supplemental material available at dmd.aspetjournals.org.
Molanaei et al., 2012), and several cytokines [interleukin (IL)-1 $\beta$, IL- $1 \alpha$, IL-6, and tumor necrosis factor (TNF)- $\alpha$ ] have been reported to downregulate activity of multiple P450 enzymes in rodent and cell models (Renton, 2005; Aitken and Morgan, 2007). Cytokines, which are formed as part of the immune response during various inflammatory conditions, have received the most attention as potential enzyme suppressors (Christensen and Hermann, 2012; Christmas, 2015), but other mediators of immune responses could be of potential importance for suppressed P450 metabolism as well.

Midazolam is considered the gold standard probe drug for CYP3A4 phenotyping. However, $4 \beta$-hydroxycholesterol ( $4 \beta \mathrm{OHC})$, a cholesterol metabolite mainly formed by CYP3A4 (Bodin et al., 2002), has attracted great interest for several years as an alternative biomarker (Bodin et al., 2001; Diczfalusy et al., 2008). Compared with midazolam, an advantage of $4 \beta \mathrm{OHC}$ is its status as an endogenous marker, and the level of $4 \beta \mathrm{OHC}$ has in previous studies been shown to respond to both inducers and inhibitors of CYP3A4 (Josephson et al., 2008; Hole et al., 2017). Moreover, $4 \beta \mathrm{OHC}$ seems to reflect both hepatic and intestinal CYP3A4 phenotypes (Gjestad et al., 2016), and the reported correlations between $4 \beta \mathrm{OHC}$ levels and concentrations of drugs metabolized by CYP3A4 (Vanhove et al., 2016; Gjestad et al., 2017) supports its usefulness as a CYP3A4 biomarker.

A previous study showed that $4 \beta \mathrm{OHC}$ levels in patients with rheumatoid arthritis (RA) were significantly lower $(\sim 20 \%)$ compared

ABBREVIATIONS: CRP, C-reactive protein; IL, interleukin; $4 \beta \mathrm{OHC}, 4 \beta$-hydroxycholesterol; P450, cytochrome P450; ra, receptor antagonist; RA, rheumatoid arthritis; Th, T-helper; TNF, tumor necrosis factor. 
with healthy controls, both before and after initiation of biologic diseasemodifying antirheumatic drugs (Wollmann et al., 2017). To obtain knowledge on inflammatory mediators possibly supressing CYP3A4 metabolism in RA patients, the aim of this pilot study was to investigate the associations between serum levels of $4 \beta \mathrm{OHC}$ and cytokines representing different immune responses in patients treated with TNF- $\alpha$ inhibitors in a real-life clinical setting.

\section{Materials and Methods}

Patients. RA patients $(n=31)$ starting treatment with TNF- $\alpha$ inhibitors were included from the Norwegian disease-modifying antirheumatic drug study (ClinicalTrials.gov; identifier NCT01581294). The study protocol, which was previously described in detail (Wollmann et al., 2017), included serum samples collected at baseline (before start of TNF- $\alpha$ inhibitor treatment) and after 3 months of treatment. In the present study, these samples were used for analysis of cytokines, $4 \beta \mathrm{OHC}$, and total cholesterol. The serum samples were stored at $-20^{\circ} \mathrm{C}$ until analysis. Information about C-reactive protein (CRP) concentrations, disease activity score 28 (Prevoo et al., 1995), and clinical disease activity index (Aletaha et al., 2005) were available from the Norwegian disease-modifying antirheumatic drug study database. This database also provided details about gender, age, and disease duration. The study was carried out in accordance with the Declaration of Helsinki and approved by the Regional Committee for Medicinal and Health Research Ethics and the Hospital Investigational Review Board.

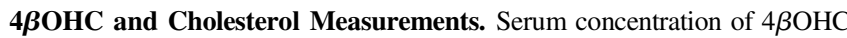
was determined by an ultra-performance liquid chromatography-tandem mass spectrometry method previously described in detail (Gjestad et al., 2016). Briefly, $10 \mu \mathrm{l}$ purified sample, also containing the internal standard (deuterium-labeled $4 \beta \mathrm{OHC} ; 4 \beta \mathrm{OHC}-\mathrm{d} 7)$, was analyzed on a Waters Acquity Quattro Micro ultraperformance liquid chromatography-tandem mass spectrometry system (Waters, Milford, MA) with a Waters Acquity ultra-performance liquid chromatography BEH Shield RP18 column $(1.7 \mu \mathrm{m}, 1 \times 100 \mathrm{~mm})$. A gradient elution with a mix of water and methanol (85\%-95\%) was used as the mobile phase for chromatographic separation, while tandem mass spectrometry detection was obtained by an atmospheric pressure chemical ionization probe operated in positive mode. The transitions that were used were $385 \rightarrow 367$ and $392 \rightarrow 374$ for $4 \beta \mathrm{OHC}$ and $4 \beta \mathrm{OHC}-\mathrm{d} 7$, respectively. Total run time was 10 minutes and the retention time of $4 \beta \mathrm{OHC}$ was 3 minutes.

The concentration data of $4 \beta \mathrm{OHC}$ applied in the present investigation was also included in a recent investigation (Wollmann et al., 2017), where patients treated with TNF- $\alpha$ inhibitors comprised the major subgroup. In this follow-up project, additional concentration measurements of cholesterol, the precursor of $4 \beta \mathrm{OHC}$, and analyses of a range of different cytokines were performed in the same serum samples. Total serum cholesterol was determined by a standard enzymatic method based on hydrolysis of cholesterol esters to free cholesterol (Roche Diagnostics $\mathrm{GmbH}$, Mannheim, Germany).

Quantification of Cytokines. Serum concentration of the following 21 cytokines was measured in the patients' samples: CCL2, CCL3, CXCL8, granulocyte colony-stimulating factor, granulocyte-macrophage colony-stimulating factor, interferon $\gamma$, IL-1 $\beta$, IL-1 receptor antagonist (ra), IL-2, IL-4, IL-5, IL-6, IL-7, IL-10, IL-12, IL-13, IL-15, IL-17A, IL-18, IL-23, and TNF- $\alpha$. IL-23 was analyzed as a single-plex assay, whereas the remaining 20 cytokines were analyzed as a multiplex assay, both bead-based immunoassays (Bio-Rad, Hercules, CA) based on xMAP technology (Luminex, Austin, TX). The cytokines were selected based on representing a broad repertoire of immune responses, including T-helper (Th)1, Th2, Th17, and macrophage activation, and on the previously documented role in the immunology in RA and response to TNF- $\alpha$ inhibition. The manufacturer's analytical protocol was followed. For values below the lower limit of quantification, samples with fluorescence values above blank were set to $50 \%$ of the lower limit of quantification, while samples with fluorescence values at blank or lower were regarded as $0 \mathrm{pg} / \mathrm{ml}$.

Statistics. The potential associations between the various cytokines, CRP, and $4 \beta \mathrm{OHC}$ levels were assessed using Spearman's rank correlation tests both at baseline and at 3 months following treatment. These correlations were performed both for the whole patient population and in females only. The Pearson's correlation test was used to investigate the association between unadjusted and cholesterol-adjusted $4 \beta \mathrm{OHC}$ levels, while the Wilcoxon signed rank test was used to compare biomarker levels after versus before initiation of TNF- $\alpha$ inhibitor treatment. All statistical analyses were conducted using GraphPad Prism version 6 (GraphPad Software, Inc., San Diego, CA). In all tests, $P<0.05$ was considered significant.

\section{Results}

Patient Characteristics. Demographic and clinical characteristics of the patients, including CRP levels and composite disease activity measures (disease activity score 28 and clinical disease activity index) are summarized in Table 1. Regarding the various TNF- $\alpha$ inhibitors that the patients were treated with, the patient majority $(n=21)$ started treatment with certolizumab pegol. Five and four patients started treatment with golimumab and etanercept, respectively, whereas one patient started treatment with adalimumab.

After 3 months of treatment with TNF- $\alpha$ inhibitors the median CRP level was $<5 \mathrm{mg} / \mathrm{l}$ (median $2 \mathrm{mg} / \mathrm{l}$, range $1-63 \mathrm{mg} / \mathrm{l}$ ), which is the reference range of normal. The low inflammation activity was also reflected by the modest scores in composite disease activity measures (Table 1). CRP values were lower than before treatment with TNF- $\alpha$ inhibitors, but the difference was not significant (median $5 \mathrm{mg} / \mathrm{l}$, range, $1-52 \mathrm{mg} / \mathrm{l}, P>0.2$ ). Three months of treatment with TNF- $\alpha$ inhibitors did not alter the $4 \beta \mathrm{OHC}$ levels in the patients (median $49 \mathrm{vs.} 52 \mathrm{nmol} / \mathrm{l}$; $P>0.9$, Wilcoxon signed rank test).

Correlation Analyses. Spearman's correlation $r$ and $P$ values for the correlation analyses between all of the cytokines, $4 \beta \mathrm{OHC}$, and CRP after 3 months of treatment with TNF- $\alpha$ inhibitors are presented in Table 2. More than $50 \%$ of the samples had undetectable fluorescence values of CCL3, and this cytokine was therefore excluded from the statistical analyses.

Significant negative correlations were observed between $4 \beta \mathrm{OHC}$ and IL-1ra (Fig. 1A, Spearman $r=-0.408, P=0.023$ ), IL-6 (Fig. 1B, $r=-0.410, P=0.022$ ), and CXCL8 (Fig. $1 \mathrm{C}, r=-0.403, P=0.025$ ). Correlations between $4 \beta \mathrm{OHC}$ and the remaining cytokines were not statistically significant $(P$ values were $>0.2)$.

IL-6 and CXCL8 were also correlated positively with CRP, i.e., IL-6 versus CRP $(r=0.541, P=0.002)$ and CXCL8 versus CRP $(r=0.402, P$ $=0.025)$. A positive, nonsignificant correlation between IL-1ra and CRP was also observed ( $r=0.340, P=0.061$ ). Prior to TNF- $\alpha$ inhibitor treatment, none of the analyzed cytokines were correlated with CYP3A4 phenotype $(P>0.1$; Supplemental Table 1$)$. None of the cytokines were correlated with CRP before treatment with TNF- $\alpha$ inhibitors $(P>$ 0.1 ; Supplemental Table 1), apart from IL-6 versus CRP ( $r=0.372$, $P=0.043)$.

In separate correlation analyses between $4 \beta \mathrm{OHC}$ and cytokine levels in female RA patients, the same three cytokines were significantly correlated with CYP3A4 activity during treatment with TNF- $\alpha$ inhibitors, i.e., IL-1ra $(r=-0.441, P=0.027)$, IL-6 $(r=-0.506, P=0.010)$, and CXCL8 $(r=-0.418, P=0.037)$ (Table 3$)$. IL-1ra, IL-6, and CXCL8 were also the only cytokines that were negatively correlated with $4 \beta \mathrm{OHC}$ in separate analyses of the patients treated with certolizumab pegol, which was the TNF- $\alpha$ inhibitor the majority of the patients (68\%) were treated with [IL-1ra $(r=0.631, P=0.002)$, IL-6 $(r=-0.507$, $P=0.019)$, and CXCL8 $(r=-0.461, P=0.061)]$ (Supplemental Table 2).

Unadjusted versus Cholesterol-Adjusted $4 \beta O H C$ Levels. For patients who had sufficient serum sample volumes available for measurement of both cytokine levels and total cholesterol concentration, Pearson's correlation analysis was used to investigate the correlation between unadjusted and cholesterol-adjusted $4 \beta \mathrm{OHC}$ levels both prior to and during use of TNF- $\alpha$ inhibitors. Twenty-five out of the 31 samples prior to and 28 out of the 31 samples during treatment with TNF- $\alpha$ 
TABLE 1

Patient characteristics

Baseline characteristics of the 31 patients with RA are given. The same patients were also included in a previously published paper (Wollmann et al., 2017).

\begin{tabular}{|c|c|}
\hline Characteristic & Data \\
\hline Age, years median (range) & $56(19-76)$ \\
\hline Gender (male/female) & $6 / 25$ \\
\hline $\mathrm{CRP}, \mathrm{mg} / \mathrm{l}$, median (range) & $5(1-52)$ \\
\hline $\mathrm{DAS} 28^{a}$, median (range) & $3.9(2.4-5.8)$ \\
\hline $\mathrm{CDAI}^{a}$, median (range) & $11.6(4.6-39.9)$ \\
\hline Time since RA diagnosis ${ }^{b}$, months, median (range) & $57(3-291)$ \\
\hline \multicolumn{2}{|l|}{ Synthetic DMARDs } \\
\hline Methotrexate & 26 \\
\hline Sulfasalazine & 7 \\
\hline Leflunomide & 5 \\
\hline Hydroxychloroquine & 3 \\
\hline Nonsteroidal anti-inflammatory drugs/systemic corticosteroids & $10 / 18$ \\
\hline \multicolumn{2}{|l|}{ TNF- $\alpha$ inhibitor initiated } \\
\hline Certolizumab pegol & 21 \\
\hline Golimumab & 5 \\
\hline Etanercept & 4 \\
\hline Adalimumab & 1 \\
\hline
\end{tabular}

CDAI, clinical disease activity index; DAS28, disease activity score 28; DMARD, disease modifying anti-rheumatic drug

${ }^{a}$ Disease activity score 28 available for $n=28$ and clinical disease activity index available for $n=29$.

${ }^{b}$ Time since RA diagnosis available for $n=19$.

inhibitors had sufficient serum volumes available for total cholesterol measurements. Significant, almost linear positive correlations between absolute $4 \beta \mathrm{OHC}$ (unadjusted) concentration and $4 \beta \mathrm{OHC} /$ cholesterol ratio were observed both prior to and during stable treatment with TNF$\alpha$ inhibitors (Pearson's $r>0.9 ; P<0.0001$, Supplemental Fig. 1).

\section{Discussion}

CYP3A4 metabolism is suppressed during systemic inflammation, but little is known about the potential mechanism(s) behind the reduced enzyme activity. In this study, we investigated the correlation between CYP3A4 phenotype and 21 cytokines reflecting different immune responses in RA patients, and observed significant negative correlations between CYP3A4 activity (4 $\beta$ OHC level) and IL-1ra, IL-6, and CXCL8 during treatment with TNF- $\alpha$ inhibitors. These preliminary findings might suggest that IL-1ra, IL-6, and/or CXCL8 are mediators or indirect measures of the suppressed CYP3A4 metabolism in RA patients.

The causality regarding the observed correlations is unclear, but a possible hypothesis is that one or more of the cytokines directly or indirectly suppress CYP3A4 activity by modulating levels of transcription factors in the liver (Morgan et al., 2008). Post-translational inhibition of enzyme function and/or destabilization of enzymes through formation of nitric oxide are also potential mechanisms (Morgan et al., 2008).

TABLE 2

Correlation analysis of serum levels of cytokines with $4 \beta \mathrm{OHC}$ and CRP

Estimated $\mathrm{r}$ values, 95\% confidence intervals, and $\mathrm{P}$ values from Spearman's rank correlation tests of the associations between cytokines and $4 \beta O H C$, and between cytokines and CRP during treatment with TNF- $\alpha$ inhibitors in 31 patients with RA.

\begin{tabular}{|c|c|c|c|c|}
\hline \multirow{2}{*}{ Cytokine } & \multicolumn{2}{|l|}{$4 \beta \mathrm{OHC}$} & \multicolumn{2}{|l|}{ CRP } \\
\hline & $r(95 \% \mathrm{CI})$ & $P$ value & $r(95 \% \mathrm{CI})$ & $P$ value \\
\hline CCL2 & $-0.147(-0.485$ to 0.229$)$ & 0.431 & $0.470(0.128-0.712)$ & 0.008 \\
\hline CXCL8 & $-0.403(-0.669$ to -0.046$)$ & 0.025 & $0.402(0.045-0.668)$ & 0.025 \\
\hline G-CSF & $-0.030(-0.390$ to 0.338$)$ & 0.873 & $0.085(-0.288$ to 0.435$)$ & 0.651 \\
\hline GM-CSF & $-0.136(-0.476$ to 0.240$)$ & 0.466 & $0.145(-0.231$ to 0.484$)$ & 0.436 \\
\hline IFN- $\gamma$ & $0.007(-0.357$ to 0.370$)$ & 0.968 & $0.034(-0.334$ to 0.393$)$ & 0.856 \\
\hline IL-1ra & $-0.408(-0.672$ to -0.052$)$ & 0.023 & $0.340(-0.027$ to 0.626$)$ & 0.061 \\
\hline $\mathrm{IL}-1 \beta$ & $-0.099(-0.447$ to 0.275$)$ & 0.596 & $0.087(-0.286$ to 0.437$)$ & 0.642 \\
\hline IL-2 & $-0.110(-0.456$ to 0.264$)$ & 0.555 & $0.092(-0.282$ to 0.441$)$ & 0.623 \\
\hline IL-4 & $-0.084(-0.435$ to 0.288$)$ & 0.651 & $-0.004(-0.367$ to 0.361$)$ & 0.985 \\
\hline IL-5 & $-0.137(-0.477$ to 0.239$)$ & 0.464 & $0.180(-0.197$ to 0.510$)$ & 0.333 \\
\hline IL-6 & $-0.410(-0.673$ to -0.054$)$ & 0.022 & $0.541(0.221-0.756)$ & 0.002 \\
\hline IL-7 & $-0.193(-0.520$ to 0.184$)$ & 0.299 & $0.214(-0.163$ to 0.536$)$ & 0.249 \\
\hline IL-10 & $0.071(-0.301$ to 0.424$)$ & 0.704 & $0.087(-0.286$ to 0.437$)$ & 0.642 \\
\hline IL-12 & $-0.154(-0.491$ to 0.222$)$ & 0.407 & $0.169(-0.207$ to 0.502$)$ & 0.362 \\
\hline IL-13 & $-0.099(-0.447$ to 0.275$)$ & 0.596 & $0.087(-0.286$ to 0.437$)$ & 0.642 \\
\hline IL-15 & $-0.029(-0.389$ to 0.338$)$ & 0.876 & $0.059(-0.312$ to 0.414$)$ & 0.753 \\
\hline IL-17A & $-0.073(-0.426$ to 0.299$)$ & 0.695 & $-0.005(-0.368$ to 0.359$)$ & 0.978 \\
\hline IL-18 & $-0.079(-0.431$ to 0.293$)$ & 0.672 & $0.165(-0.211$ to 0.499$)$ & 0.374 \\
\hline IL-23 & $0.039(-0.329$ to 0.398$)$ & 0.833 & $-0.020(-0.381$ to 0.346$)$ & 0.914 \\
\hline TNF- $\alpha$ & $-0.109(-0.455$ to 0.266$)$ & 0.561 & $0.325(-0.044$ to 0.616$)$ & 0.075 \\
\hline
\end{tabular}

CI, confidence interval; G-CSF, granulocyte colony-stimulating factor; GM-CSF, granulocyte-macrophage colony-stimulating factor; IFN- $\gamma$, interferon $\gamma$. 

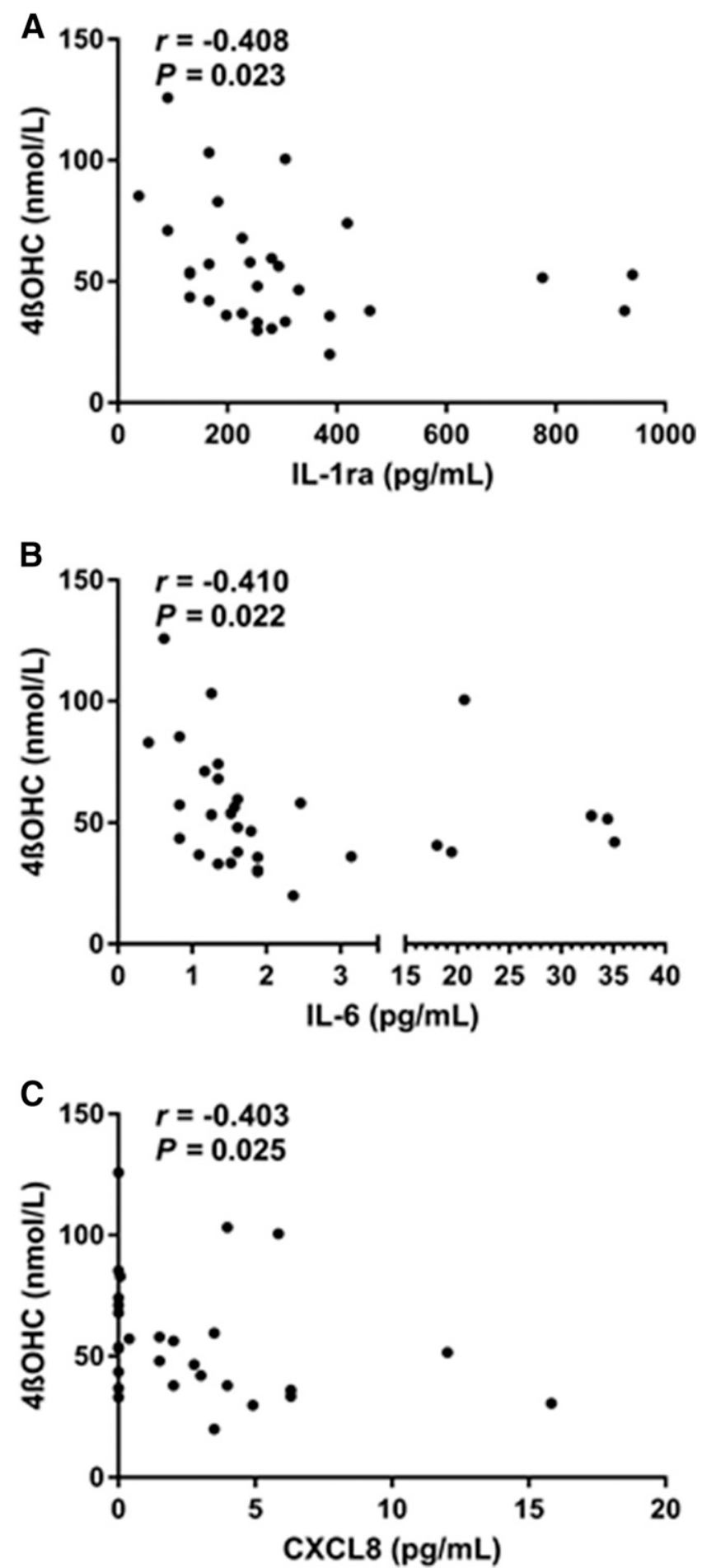

Fig. 1. Correlations between $4 \beta \mathrm{OHC}$ levels and levels of interleukin (IL)-1ra (A), IL-6 (B), and CXCL8 (C) during treatment with TNF- $\alpha$ inhibitors in 31 patients with RA. Estimated $r$ and $P$ values from Spearman's rank correlation test are added in each illustration. In (A and $\mathrm{C}$ ) one and two data points are outside the respective axis limits.

Among the cytokines studied in relation to suppression of activity of drug-metabolizing enzymes, IL-6 has been the subject of most interest. Suppression of CYP3A mRNA by IL-6 has been reported in several in vitro studies (Abdel-Razzak et al., 1993; Sunman et al., 2004; Aitken and Morgan, 2007; Dickmann et al., 2011). Aitken and Morgan (2007) showed that IL-6 reduced the mRNA expression of CYP3A4 and
CYP3A4 protein level to $5 \%$ and $50 \%$ of the control, respectively, in human hepatocytes; mRNA expression of other isoenzymes was also tested in the same study, and IL-6 was the only cytokine or molecule associated with immune response, which consistently showed a reduction in all isoenzymes (apart from CYP2C18).

No studies have thus far reported a correlation between levels of IL-6 and CYP3A4 metabolism in patients with chronic inflammation. However, indirect evidence demonstrating the importance of IL-6 in CYP3A4 activity in vivo has been obtained in two studies measuring metabolism after versus before use of IL-6 inhibitors (Schmitt et al., 2011; Zhuang et al., 2015). In the two small studies with RA patients $(n=12)$, initiation of treatment with IL-6 inhibitors tocilizumab and sirukumab resulted in significant lower exposure (area under the curve) of CYP3A4 substrates simvastatin and midazolam, respectively. Together with our novel data, these studies support the hypothesis that IL-6 is a central cytokine for regulation of CYP3A4 activity in patients with inflammation-driven diseases.

The two other cytokines correlating with CYP3A4 activity in our study (i.e., IL-1 ra and CXCL8) have not previously been implicated in a decline in CYP3A4 activity in RA patients. However, in vitro studies have demonstrated that IL-1 ( $\alpha$ and $\beta$ ) reduces CYP3A expression in human hepatocytes (Abdel-Razzak et al., 1993; Sunman et al., 2004; Aitken and Morgan, 2007), and this effect has been shown to be significantly reversed by IL-1ra in human hepatoma cell lines (Mimura et al., 2015). IL-1 ra is a competitive antagonist to the IL-1 receptor, thereby blocking the inflammatory properties of IL- $1 \alpha$ and IL- $1 \beta$, and levels of these three cytokines are often increased simultaneously (Arend, 2002). Although the effect of CXCL8 on CYP3A4 activity has not been investigated, these in vitro studies might explain why levels of IL-1ra were found to be negatively correlated with $4 \beta \mathrm{OHC}$ in the present study. Nevertheless, future studies are required to clarify the roles of IL-1ra and CXCL8 in regulating CYP3A4 phenotype in vivo.

IL-6, CXCL8, and IL-1 $\beta$ are all cytokines reflecting Th17 cell responses (Tesmer et al., 2008), which are considered to be central in mediating the disease process in RA (McInnes and Schett, 2007). IL-6, IL- $1 \beta$, TNF- $\alpha$, and IL-23 are key inflammation-promoting cytokines released as part of the Th17 cell immune response, while induction of CXCL8, among other chemokines, leads to recruitment of $\mathrm{T}$ cells, B cells, monocytes, and neutrophils to the inflamed joint (Tesmer et al., 2008). Thus, our results indicate a role for Th17 cells in suppression of CYP3A4 activity.

IL-1ra, IL-6, and CXCL8 were also the only cytokines that were correlated with $4 \beta \mathrm{OHC}$ during TNF- $\alpha$ inhibitor treatment in the separate correlation analysis of the female RA patients. Interestingly, the correlations were stronger in females than in the whole population. The reason for this is unclear, but a factor of importance could be that females generally exhibit higher CYP3A4 activities than males (Gjestad et al., 2017; Hole et al., 2017). Additionally, more cytokines were positively correlated with CRP when analyzing the female RA patients than in the analysis of the whole population. Thus, it is crucial that future studies investigating the effects of inflammation on CYP3A4 metabolism include sex as variable.

Previous in vitro studies have reported downregulated expression of CYP3A4 by TNF- $\alpha$ (Aitken and Morgan, 2007; Mimura et al., 2015). However, this was not supported by our study, where we did not observe any correlations between TNF- $\alpha$ and $4 \beta \mathrm{OHC}$, neither prior to or after 3 months of treatment with TNF- $\alpha$ inhibitors. Furthermore, initiation of treatment with TNF- $\alpha$ inhibitors did not alter CYP3A4 phenotype after 3 months. The discrepancy between the results in our study and the in vitro studies might be explained by the fact that the most pronounced effects on CYP3A4 expression in in vitro studies were shown for IL-1, IL-6, and lipopolysaccharide (Aitken and Morgan, 2007; Mimura et al., 2015), 
TABLE 3

Correlation analysis of serum levels of cytokines with $4 \beta \mathrm{OHC}$ and CRP in female RA patients

Estimated $\mathrm{r}$ values, 95\% confidence intervals, and $\mathrm{P}$ values from Spearman's rank correlation tests of the associations between cytokines and $4 \beta O H C$, and between cytokines and CRP during treatment with TNF- $\alpha$ inhibitors in 25 female patients with RA.

\begin{tabular}{|c|c|c|c|c|}
\hline \multirow{2}{*}{ Cytokine } & \multicolumn{2}{|l|}{$4 \beta \mathrm{OHC}$} & \multicolumn{2}{|l|}{ CRP } \\
\hline & $r(95 \% \mathrm{CI})$ & $P$ value & $r(95 \% \mathrm{CI})$ & $P$ value \\
\hline CXCL8 & $-0.418(-0.704$ to -0.015$)$ & 0.037 & $0.577(0.224-0.796)$ & 0.003 \\
\hline IL-1ra & $-0.441(-0.718$ to -0.044$)$ & 0.027 & $0.497(0.115-0.751)$ & 0.011 \\
\hline IL-2 & $-0.250(-0.595$ to 0.173$)$ & 0.228 & $0.399(-0.007$ to 0.693$)$ & 0.048 \\
\hline IL-6 & $-0.506(-0.756$ to -0.126$)$ & 0.010 & $0.764(0.519-0.893)$ & $<0.0001$ \\
\hline IL-7 & $-0.250(-0.595$ to 0.173$)$ & 0.228 & $0.417(0.014-0.704)$ & 0.038 \\
\hline TNF- $\alpha$ & $-0.162(-0.533$ to 0.261$)$ & 0.439 & $0.533(0.163-0.772)$ & 0.006 \\
\hline
\end{tabular}

CI, confidence interval.

but further studies are needed to examine the potential roles of TNF- $\alpha$ and TNF- $\alpha$ inhibition in CYP3A4 expression in RA patients.

During treatment with TNF- $\alpha$ inhibitors, $4 \beta \mathrm{OHC}$ and CRP levels were significantly negatively correlated, as also reported in a previous study (Björkhem-Bergman et al., 2013). In our patient population, IL-6 and CXCL8 $(P<0.05)$, and IL-1ra $(P=0.061)$ were positively correlated with CRP. Thus, we consider it likely that CRP reflects levels of these cytokines in the patient population rather than mediating the downregulation of CYP3A4 activity per se. This is supported by the fact that levels of the cytokine CCL2 significantly correlated with CRP but not with $4 \beta \mathrm{OHC}$.

Prior to TNF- $\alpha$ inhibitor treatment, none of the analyzed cytokines were correlated with CYP3A4 phenotype. The RA patients constitute, at this time point, a quite heterogeneous patient population due to variability in disease state and various anti-inflammatory treatment regiments prior to starting TNF- $\alpha$ inhibitor treatment in this study. This heterogeneity is a complicating factor when investigating correlations between CYP3A4 activity and inflammation markers, and we therefore focused mainly on the correlations between cytokine levels and CYP3A4 phenotype during treatment with TNF- $\alpha$ inhibitors in the current study. In future studies it is important to examine the correlations between CYP3A4 and these cytokines in a larger population, with newly diagnosed patients, and over a longer time span after initiation of treatment.

In this study, we examined the correlations between cytokine levels and CYP3A4 activity, an important drug-metabolizing enzyme with extensive interindividual variability in phenotype. However, since systemic inflammation has shown to downregulate multiple P450 enzymes (Christensen and Hermann, 2012), it is important that the impact of inflammation on other P450 enzymes is examined. A combination of a cocktail approach, which enables multiple enzymes to be tested at once through concurrent administration of several probe drugs, and multiplex immunoassays, as used in this study, would allow a high-throughput method for investigation of the immune responses involved in suppression of P450 phenotypes.

Limitations of the current study include the heterogeneity of the patient population in terms of variable time of RA diagnosis, variability in disease activity and comorbidity, and different RA treatment regimens and non-RA comedication, which may affect levels of both $4 \beta \mathrm{OHC}$ and cytokines. Another possible limitation may be the increase likelihood of type I errors since multiple testing was performed. In addition, preanalytical sample handling procedures have been shown to affect CXCL8 levels in serum samples prior to analysis and this could have affected the reliability of the CXCL8 measurements in our study (Skogstrand et al., 2008; de Jager et al., 2009). However, it is unlikely that systematic differences in sample handling procedures have affected our results.
In conclusion, this is the first study to examine the associations between serum levels of a range of cytokines and CYP3A4 metabolism in patients with chronic inflammation. The significant and negative correlations of $4 \beta \mathrm{OHC}$ levels with IL-1ra, IL-6, and CXCL8 suggest that these cytokines and associated immune responses might be associated with suppressed CYP3A4 metabolism in RA patients. These preliminary findings imply that these cytokines may be mediators or indirect measures of the suppressed CYP3A4 metabolism in RA patients with chronic inflammation; however, additional studies are required both to confirm these findings and to examine the possible molecular mechanisms behind our observations.

\section{Acknowledgments}

We thank Gro Jensen and Siri Beisvåg Rom at Department of Biochemistry, Diakonhjemmet Hospital, for excellent technical assistance on the cytokine analyses.

\section{Authorship Contributions}

Participated in research design: Wollmann, Syversen, Vistnes, Lie, Molden. Conducted experiments: Wollmann, Vistnes, Mehus.

Performed data analysis: Wollmann, Syversen, Vistnes, Molden.

Wrote or contributed to the writing of the manuscript: Wollmann, Syversen, Vistnes, Lie, Molden.

\section{References}

Abdel-Razzak Z, Loyer P, Fautrel A, Gautier JC, Corcos L, Turlin B, Beaune P, and Guillouzo A (1993) Cytokines down-regulate expression of major cytochrome P-450 enzymes in adult human hepatocytes in primary culture. Mol Pharmacol 44:707-715.

Aitken AE and Morgan ET (2007) Gene-specific effects of inflammatory cytokines on cytochrome P450 2C, 2B6 and 3A4 mRNA levels in human hepatocytes. Drug Metab Dispos 35:1687-1693. Aletaha D, Nell VP, Stamm T, Uffmann M, Pflugbeil S, Machold K, and Smolen JS (2005) Acute phase reactants add little to composite disease activity indices for rheumatoid arthritis: validation of a clinical activity score. Arthritis Res Ther 7:R796-R806.

Arend WP (2002) The balance between IL-1 and IL-1Ra in disease. Cytokine Growth Factor Rev 13:323-340.

Björkhem-Bergman L, Nylén H, Norlin AC, Lindh JD, Ekström L, Eliasson E, Bergman P, and Diczfalusy U (2013) Serum levels of 25-hydroxyvitamin D and the CYP3A biomarker $4 \beta$ hydroxycholesterol in a high-dose vitamin D supplementation study. Drug Metab Dispos 41: 704-708.

Bodin K, Andersson U, Rystedt E, Ellis E, Norlin M, Pikuleva I, Eggertsen G, Björkhem I, and Diczfalusy U (2002) Metabolism of $4 \beta$-hydroxycholesterol in humans. $J$ Biol Chem 277: 31534-31540.

Bodin K, Bretillon L, Aden Y, Bertilsson L, Broomé U, Einarsson C, and Diczfalusy U (2001) Antiepileptic drugs increase plasma levels of $4 \beta$-hydroxycholesterol in humans: evidence for involvement of cytochrome P450 3A4. J Biol Chem 276:38685-38689.

Christensen H and Hermann M (2012) Immunological response as a source to variability in drug metabolism and transport. Front Pharmacol 3:8.

Christmas P (2015) Role of cytochrome P450s in inflammation. Adv Pharmacol 74:163-192

de Jager W, Bourcier K, Rijkers GT, Prakken BJ, and Seyfert-Margolis V (2009) Prerequisites for cytokine measurements in clinical trials with multiplex immunoassays. BMC Immunol 10:52.

Dickmann LJ, Patel SK, Rock DA, Wienkers LC, and Slatter JG (2011) Effects of interleukin-6 (IL-6) and an anti-IL-6 monoclonal antibody on drug-metabolizing enzymes in human hepatocyte culture. Drug Metab Dispos 39:1415-1422.

Diczfalusy U, Miura J, Roh HK, Mirghani RA, Sayi J, Larsson H, Bodin KG, Allqvist A, Jande M, Kim JW, et al. (2008) 4 $\beta$-Hydroxycholesterol is a new endogenous CYP3A marker: relationship to CYP3A5 genotype, quinine 3-hydroxylation and sex in Koreans, Swedes and Tanzanians. Pharmacogenet Genomics 18:201-208. 
Gjestad C, Haslemo T, Andreassen OA, and Molden E (2017) 4 $\beta$-Hydroxycholesterol level significantly correlates with steady-state serum concentration of the CYP3A4 substrate quetiapine in psychiatric patients. Br J Clin Pharmacol 83:2398-2405.

Gjestad C, Huynh DK, Haslemo T, and Molden E (2016) $4 \beta$-hydroxycholesterol correlates with dose but not steady-state concentration of carbamazepine: indication of intestinal CYP3A in biomarker formation? Br J Clin Pharmacol 81:269-276.

He P, Court MH, Greenblatt DJ, and Von Moltke LL (2005) Genotype-phenotype associations of cytochrome P450 3A4 and 3A5 polymorphism with midazolam clearance in vivo. Clin Phar macol Ther 77:373-387.

Hole K, Gjestad C, Heitmann KM, Haslemo T, Molden E, and Bremer S (2017) Impact of genetic and nongenetic factors on interindividual variability in $4 \beta$-hydroxycholesterol concentration. Eur J Clin Pharmacol 73:317-324.

Josephson F, Bertilsson L, Böttiger Y, Flamholc L, Gisslén M, Ormaasen V, Sönnerborg A, and Diczfalusy U (2008) CYP3A induction and inhibition by different antiretroviral regimens reflected by changes in plasma $4 \beta$-hydroxycholesterol levels. Eur J Clin Pharmacol 64: $775-781$.

Klein K and Zanger UM (2013) Pharmacogenomics of cytochrome P450 3A4: recent progress toward the "missing heritability" problem. Front Genet 4:12.

Mayo PR, Skeith K, Russell AS, and Jamali F (2000) Decreased dromotropic response to verapamil despite pronounced increased drug concentration in rheumatoid arthritis. Br J Clin Pharmaco 50:605-613.

McInnes IB and Schett G (2007) Cytokines in the pathogenesis of rheumatoid arthritis. Nat Rev Immunol 7:429-442.

Mimura H, Kobayashi K, Xu L, Hashimoto M, Ejiri Y, Hosoda M, and Chiba K (2015) Effects of cytokines on CYP3A4 expression and reversal of the effects by anti-cytokine agents in the threedimensionally cultured human hepatoma cell line FLC-4. Drug Metab Pharmacokinet 30: $105-110$

Molanaei H, Stenvinkel P, Qureshi AR, Carrero JJ, Heimbürger O, Lindholm B, Diczfalusy U, Odar-Cederlöf I, and Bertilsson L (2012) Metabolism of alprazolam (a marker of CYP3A4) in hemodialysis patients with persistent inflammation. Eur J Clin Pharmacol 68:571-577.

Morgan ET, Goralski KB, Piquette-Miller M, Renton KW, Robertson GR, Chaluvadi MR, Charles KA, Clarke SJ, Kacevska M, Liddle C, et al. (2008) Regulation of drug-metabolizing enzymes and transporters in infection, inflammation, and cancer. Drug Metab Dispos 36:205-216.

Prevoo ML, van't Hof MA, Kuper HH, van Leeuwen MA, van de Putte LB, and van Riel PL (1995) Modified disease activity scores that include twenty-eight-joint counts. Development and validation in a prospective longitudinal study of patients with rheumatoid arthritis. Arthritis Rheum 38:44-48.
Renton KW (2005) Regulation of drug metabolism and disposition during inflammation and infection. Expert Opin Drug Metab Toxicol 1:629-640.

Rivory LP, Slaviero KA, and Clarke SJ (2002) Hepatic cytochrome P450 3A drug metabolism is reduced in cancer patients who have an acute-phase response. Br J Cancer 87:277-280.

Schmitt C, Kuhn B, Zhang X, Kivitz AJ, and Grange S (2011) Disease-drug-drug interaction involving tocilizumab and simvastatin in patients with rheumatoid arthritis [published correction appears in Clin Pharmacol Ther 2011:90:479]. Clin Pharmacol Ther 89:735-740.

Skogstrand K, Ekelund CK, Thorsen P, Vogel I, Jacobsson B, Nørgaard-Pedersen B, and Hougaard DM (2008) Effects of blood sample handling procedures on measurable inflammatory markers in plasma, serum and dried blood spot samples. J Immunol Methods 336:78-84.

Sunman JA, Hawke RL, LeCluyse EL, and Kashuba ADM (2004) Kupffer cell-mediated IL-2 suppression of CYP3A activity in human hepatocytes. Drug Metab Dispos 32:359-363.

Tesmer LA, Lundy SK, Sarkar S, and Fox DA (2008) Th17 cells in human disease. Immunol Rev 223:87-113.

Ulvestad M, Skottheim IB, Jakobsen GS, Bremer S, Molden E, Asberg A, Hjelmesæth J, Andersson TB, Sandbu R, and Christensen H (2013) Impact of OATP1B1, MDR1, and CYP3A4 expression in liver and intestine on interpatient pharmacokinetic variability of atorvastatin in obese subjects. Clin Pharmacol Ther 93:275-282.

Vanhove T, de Jonge H, de Loor H, Annaert P, Diczfalusy U, and Kuypers DR (2016) Comparative performance of oral midazolam clearance and plasma $4 \beta$-hydroxycholesterol to explain interindividual variability in tacrolimus clearance. Br J Clin Pharmacol 82:1539-1549.

Wilkinson GR (2005) Drug metabolism and variability among patients in drug response. $N$ Engl J Med 352:2211-2221.

Wollmann BM, Syversen SW, Lie E, Gjestad C, Mehus LL, Olsen IC, and Molden E (2017) $4 \beta$ hydroxycholesterol level in patients with rheumatoid arthritis before vs. after initiation of bDMARDs and correlation with inflammatory state. Clin Transl Sci 10:42-49.

Zhuang Y, de Vries DE, Xu Z, Marciniak SJ, Jr, Chen D, Leon F, Davis HM, and Zhou H (2015) Evaluation of disease-mediated therapeutic protein-drug interactions between an anti-interleukin6 monoclonal antibody (sirukumab) and cytochrome P450 activities in a phase 1 study in patients with rheumatoid arthritis using a cocktail approach. J Clin Pharmacol 55:1386-1394.

Address correspondence to: Birgit M. Wollmann, Center for Psychopharmacology, PO Box 85 Vinderen, N-0319, Oslo, Norway. E-mail: birgitmalenetovik. wollmann@diakonsyk.no 